\title{
Management of Mitral Stenosis
}

\section{Wilbert S Aronow*}

Department of Medicine, Division of Cardiology, New York Medical College, Valhalla, NY, USA

\section{Medical Management}

Because rheumatic fever is the primary cause of mitral stenosis, secondary prophylaxis against group a beta-hemolytic streptococci is recommended [1]. The recommended regimen for prevention of recurrent rheumatic fever is an intramuscular injection of $1,200,000$ units of benzathiazine penicillin every 4 weeks for most patients and every 3 weeks in populations in which the incidence of rheumatic fever is very high [1]. Penicillin V $250 \mathrm{mg}$ twice daily or sulfadiazine $500 \mathrm{mg}$ to $1,000 \mathrm{mg}$ once daily dependent on weight may be used. For penicillin-allergic persons, a narrow-spectrum oral cephalosporin, oral clindamycin, or various oral macrolides or azalides should be used. Prophylaxis should continue until at least age 40 and perhaps for life in persons with increased exposure to streptococcal infections. Prophylaxis should continue even after valve surgery [1].

The current American Heart Association guidelines no longer recommend routine bacterial endocarditis prophylaxis for persons with rheumatic mitral stenosis [2]. For the patients with rheumatic heart disease in whom infective endocarditis prophylaxis remains recommended such as patients with prosthetic valves or prosthetic material used in valve repair, a drug other than penicillin is recommended to prevent infective endocarditis in patients receiving penicillin for rheumatic fever prophylaxis [2].

Patients with mitral stenosis in sinus rhythm with exertional symptoms occurring with high ventricular rates should be treated with a beta blocker, verapamil, or diltiazem. Dietary sodium restriction and diuretic therapy should be used to treat symptoms due to pulmonary congestion. Reduction of preload by oral nitrates may also be beneficial. Prolongation of the diastolic filling time by beta blockers can reduce left atrial pressure.

Treatment of an acute episode of atrial fibrillation with a rapid ventricular rate consists of anticoagulation with heparin and reduction of the ventricular rate by intravenous beta blockers, diltiazem, or verapamil and with amiodarone if these drugs cannot be used. If there is hemodynamic instability, the patient should be treated with direct-current cardioversion immediately with intravenous heparin administered before, during, and after the procedure. The ventricular rate in patients with atrial fibrillation should be controlled long-term by use of beta blockers, verapamil, diltiazem, amiodarone, or digoxin.

Class I indications for use of oral warfarin in patients with mitral stenosis are paroxysmal or chronic atrial fibrillation or a prior thromboembolic event in a patient with sinus rhythm with an International Normalized Ratio (INR) maintained between 2.0 to 3.0 or a left atrial thrombus with the INR maintained between 2.5 to 3.5 [3]. The novel anticoagulant drugs such as dabigatran, rivaroxaban, and apixaban are not approved for treatment of atrial fibrillation associated with valvular heart disease.

\section{Percutaneous Mitral Commissurotomy}

The American College of Cardiology/American Heart Association 2006 guidelines recommend that Percutaneous Mitral Balloon Valvotomy (PMBV) is effective for symptomatic patients with New York Heart Association (NYHA) functional class II, III, or IV symptoms with moderate or severe mitral stenosis (mitral valve area of $1.5 \mathrm{~cm}^{2}$ or less) with a valve morphology suitable for PMBV in the absence of left atrial thrombus or moderate to severe mitral regurgitation with a class I indication [3]. Transesophageal echocardiography should be performed with a class I indication to assess the presence or absence of left atrial thrombus and to further evaluate the severity of mitral regurgitation in patients considered for PMBV [3]. These guidelines also recommend with a class I indication that PMBV is effective for asymptomatic patients with moderate or severe mitral stenosis and a valve morphology suitable for PMBV who have a pulmonary artery systolic pressure greater than $50 \mathrm{~mm} \mathrm{Hg}$ at rest or greater than $60 \mathrm{~mm}$ $\mathrm{Hg}$ with exercise in the absence of left atrial thrombus or moderate to severe mitral regurgitation [3]. These guidelines recommend with a class IIa indication PMBV for patients with moderate or severe mitral stenosis who have a nonpliable calcified valve, have NYHA functional class III or IV symptoms, and either is not candidates for surgery or are at high risk for surgery [3]. PMBV is not indicated for patients with mild mitral stenosis and should not be performed in patients with left atrial thrombus or moderate to severe mitral regurgitation [3]. For elderly patients with symptomatic mitral stenosis who have an uncalcified mitral valve or mild calcific deposits, flexible mitral valve leaflets, and no or mild mitral regurgitation and no left atrial thrombus, PMBV is the procedure of choice [4].

The European Society of Cardiology (ESC)/European Association of Cardiothoracic Surgeons (EACTS) 2012 guidelines recommend Percutaneous Mitral Commissurotomy (PMC) in patients with mitral stenosis and a mitral valve area of $1.5 \mathrm{~cm}^{2}$ or less who are (1) symptomatic with favorable characteristics (class I indication), (2) in symptomatic patients with contraindication or high risk for surgery (class I indication), and (3) should be considered as initial treatment in symptomatic patients with an unfavorable mitral valve anatomy but without unfavorable clinical characteristics (class IIa indication) [5]. These guidelines also recommend consideration of PMC with a class IIa indication if they are asymptomatic without unfavorable characteristics and a high thromboembolic risk (previous history of thromboembolism, dense spontaneous contrast in the left atrium, recent or paroxysmal atrial fibrillation) and/or a pulmonary artery systolic pressure greater than $50 \mathrm{~mm} \mathrm{Hg}$ at rest, a need for major non-cardiac surgery, and a desire for pregnancy [5]. Contraindications to PMC include a mitral valve area greater than $1.5 \mathrm{~cm}^{2}$, left atrial thrombus, moderate or severe mitral regurgitation, severe or bicommissural calcification, absence of commissural fusion, severe concomitant aortic valve disease or severe

${ }^{*}$ Corresponding author: Wilbert S. Aronow, Cardiology Division, New York Medical College, Macy Pavilion, Room 138, Valhalla, NY 10595, USA, Tel: (914) 493-5311; Fax: (914) 235-6274; E-mail: wsaronow@aol.com

Received October 20, 2013; Accepted October 21, 2013; Published October 26 2013

Citation: Aronow WS (2013) Management of Mitral Stenosis. J Cardiovasc Dis Diagn 1: e104. doi:10.4172/2329-9517.1000e104

Copyright: (c) 2013 Aronow WS. This is an open-access article distributed under the terms of the Creative Commons Attribution License, which permits unrestricted use, distribution, and reproduction in any medium, provided the original author and source are credited. 
combined tricuspid stenosis and regurgitation, and concomitant coronary artery disease needing bypass surgery [5].

\section{Indications for Mitral Valve Surgery}

Mitral valve surgery (repair if possible) is indicated with a class I indication in patients with NYHA functional class II-IV symptoms with moderate or severe mitral stenosis when (1) PMBV is unavailable, (2) when PMBV is contraindicated because of left atrial thrombus despite adequate anticoagulation or because of moderate or severe mtral regurgitation, or (3) because the mitral valve morphology is unfavorable for PMBV in a patient with an acceptable operative risk [3]. Symptomatic patients with moderate to severe mitral stenosis with moderate or severe mitral regurgitation should receive mitral valve replacement unless mitral valve repair is possible at the time of surgery with a class I indication [3]. Mitral valve replacement should be considered in patients with severe mitral stenosis and a pulmonary artery systolic pressure greater than $60 \mathrm{~mm} \mathrm{Hg}$ at rest with NYHA functional class I or II symptoms who are not considered candidates for PMBV or surgical mitral valve repair with a class IIa indication [3]. Mitral valve repair for mitral stenosis should not be performed in patients with mild mitral stenosis (classs III indication) [3]. Closed commissurotomy should not be performed in patients undergoing mitral valve repair (class III indication). Open commissurotomy is the preferred approach [3]. In current practice, surgery for mitral stenosis is approximately $95 \%$ valve replacement because of elderly presentation and unfavorable characteristics for valve repair. Consider open mitral commissurotomy if the mitral valve is flexible enough to open when the commissures are released, mitral regurgitation is minimal, the subvalvular apparatus is obstructive, and there is a thrombus in the left atrium, especially in the body of the left atrium after 2 to 3 months of adequate anticogulation therapy [4].

For a mechanical mitral prosthesis, the INR should be maintained between 2.5 to 3.5 [3]. For a bioprosthetic mitral valve, aspirin 75 to $100 \mathrm{mg}$ should be administered daily if there are no risk factors [3]. If atrial fibrillation, left ventricular dysfunction, prior thromboembolism, or a hypercoagulable state is present with a bioprosthetic mitral valve, warfarin should be administered to maintain an INR between 2.5 to 3.5. None of the novel oral anticoagulants approved for non-valvular atrial fibrillation should be used.

\section{Pregnancy}

Mitral stenosis is the most common valvular lesion in pregnant women. The management of mitral stenosis in pregnant women is discussed extensively elsewhere [3,6-8].

\section{References}

1. Gerber MA, Baltimore RS, Eaton CB, Gewitz M, Rowley AH, et al. (2009) Prevention of rheumatic fever and diagnosis and treatment of acute Streptococcal pharyngitis: a scientific statement from the American Heart Association Rheumatic Fever, Endocarditis, and Kawasaki Disease Committee of the Council on Cardiovascular Disease in the Young, the Interdisciplinary Council on Functional Genomics and Translational Biology, and the interdisciplinary Council on Quality of Care and Outcomes Research: endorsed by the American Academy of Pediatrics. Circulation 119: 1541-1551.

2. Wilson W, Taubert KA, Gewitz, Lockhart PB, Baddour LM, et al. (2007) Prevention of infective endocarditis: guidelines from the American Heart association: a guideline from the American Heart Association Rheumatic Fever Enocarditis, and Kawasaki Disease Committee, Council on Cardiovascular Disease in the Yung, and the Council on clinical cardiology, Council on Cardiovascular Surgery and Anesthesia, and the Quality of Care and Outcomes Research Interdisciplinary Working Group. Circulation 116: 1736-1754.

3. Bonow RO, Carabello BA, Chatterjee K, de Leon AC, Jr, Faxon DP, et al (2006) ACC/AHA 2006 practice guidelines for the management of patients with valvular heart disease. Executive Summary. A Report of the American College of Cardiology /American Heart Association task force on Practice Guidelines (Writing Committee to Revise the 1988 Guidelines for the Management of Patients With Valvular Heart Disease). Developed in collaboration with the Society of Cardiovascular Anesthesiologists. Endorsed by the Society for Cardiovascular Angiography and Interventions and the Society of Thoracic Surgeons. J Am Coll Cardiol 48: 598-675.

4. Cheitlin M, Aronow WS (2013) Mitral regurgitation, mitral stenosis, and mitra annular calcification in the elderly. In: Aronow WS, Fleg J, Rich MW (Eds) Tresch and Aronow's Cardiovascular Disease in the Elderly ( $5^{\text {th }}$ edn), Boca Raton, CRC press, London, New York, 374-412.

5. Vahanian A, Alfieri O, Andreotti F, Antunes MJ, Baron-Esquivias G, et al (2012) Guidelines on the management of valvular heart disease. The Joint Task Force on the Management of Valvular Heart Disease of the European Society of Cardiology (ESC) and the European Association for Cardio-Thoracic Surgery (EACTS). Eur Heart J 33: 2451-2496.

6. Elkayam U, Bitar F (2005) Valvular heart disease and pregnancy part I: native valves. J Am Coll Cardiol 46: 223-230.

7. Elkayam U, Bitar F (2005) Valvular heart disease and pregnancy part II: prosthetic valves. J Am Coll Cardiol 46: 403-410.

8. Regitz-Zagrosek V, Lundqvist $C B$, Borghi $C$, Cifkova R, Ferreira R, et al (2011) ESC guidelines on the management of cardiovascular diseases during pregnancy: The Task force on the Management of Cardiovascular Diseases during Pregnancy of the European Society of Cardiology (ESC). Eur Heart J 32: 3147-3197. 